\title{
PERFIL DOS PACIENTES ATENDIDOS NA UNIDADE PRONTO ATENDIMENTO, JARDIM VENEZA, CASCAVEL- PR
}

\section{PROFILE OF PATIENTS ATTENDED IN EMERGENCY CARE UNIT, JARDIM VENEZA, CASCAVEL-PR}

\author{
Yohana Vitória de Matos ${ }^{1}$, Daiane Breda²
}

\begin{abstract}
${ }^{1}$ Acadêmica do oitavo período do curso de Medicina do Centro Universitário Fundação Assis Gurgacz. ${ }^{2}$ Médica. Mestre em Saúde Pública pela Universidade Federal de Santa Catarina. Docente do Centro Universitário Fundação Assis Gurgacz. Especialização na área de Medicina do Trabalho.

* Autor correspondente: yohanaa_vitoria@hotmail.com, https://orcid.org/0000-0002-4536-5586
\end{abstract}

DOI: $10.35984 /$ fjh.v2i1.164

\section{RESUMO}

As Unidades de Pronto Atendimento têm por objetivo prestar atendimentos às urgências e emergências, porém têm se tornado uma porta de entrada aos usuários com queixas crônicas e sociais. O presente estudo objetivou analisar o perfil dos pacientes de acordo com sexo e idade, caracterizar a demanda da unidade e verificar o manejo e desfecho dos mesmos. Tratase de um estudo descritivo-observacional, realizado de maneira retrospectiva, com abordagem quantitativa, desenvolvido com dados coletados via software-IPM e dados previamente tabulados fornecidos pelo SAME da UPA Veneza do município de Cascavel-PR. A amostra válida do estudo foi de 5.483 pacientes que realizaram a procura espontânea pela unidade no mês de maio de 2019, sendo esses, majoritariamente, do sexo feminino, jovens, entre 21 a 30 anos, com queixas pouco urgentes. Constatou-se também que o horário de maior demanda coincide com o horário de funcionamento das UBSs, tal resultado pode ocorrer devido ao fácil acesso e à resolubilidade fornecida. Conclui-se que, com atendimento 24 horas junto à dificuldade de acesso às consultas nas Unidades Básicas de Saúde e havendo agilidade na solução dos problemas de saúde, grande parte da população acaba buscando atendimento nas UPAs, mesmo quando esses problemas não representam grande complexidade.

Palavras-chave: Unidade de Pronto Atendimento, urgência, emergência, pacientes.

\begin{abstract}
The Emergency Care Units have for aim to provide services to the urgency and emergence, however they have when if an entrance door was made to the users with chronic and social complaints. The present study aimed to analyze the profile of patients according to sex and age, to characterize the demand of the unit and verify the management and outcome of the unit. This is a descriptive-observational study, carried out retrospectively, with a quantitative approach, it developed with data collected via software-IPM and previously tabulated data provided by the SAME of UPA Veneza in the municipality of Cascavel-PR. The valid sample of the study was 5,483 patients who underwent spontaneous search for the unit in May 2019, being mostly female, young people, between 21 and 30 years old, with in urgent complaints. It was also found that the most demanding time coincides with the opening hours of the UBSs, this result may occur due to the easy access and resolvability provided. It is concluded that, with 24-hour care regarding the difficulty of accessing consultations in the Basic Health Units and with agility in solving health problems, a large part of the population finishes looking for service in the UPAs, even when these problems do not represent great complexity.
\end{abstract}

Key words: Emergency Care Unit, urgency, emergence, patients. 


\section{INTRODUÇÃO}

As Unidades de Pronto Atendimento (UPAs) estão de acordo com a Política Nacional de Urgências e Emergências (PNAU), publicada pelo Ministério da Saúde em 2003 (HERNANDEZ, 2018). Efetuou-se também a publicação da Portaria no 10, no ano de 2017, na qual essa política foi redefinida, estabelecendo assim o objetivo das UPAs de integrarem a rede de atenção às urgências (BRASIL, 2017). As UPAs visam estruturar e organizar a rede de urgências e emergências do país, e estão distribuídas em pontos estratégicos da cidade, de forma que seja possível formar a Rede de Atenção às Urgências, conforme previsto na PNAU (BRASIL, 2011).

As UPAs $24 \mathrm{~h}$ são instituições de saúde de complexidade intermediária, que ficam entre as Unidades Básicas de Saúde (UBSs), Unidade de Saúde da Família (USF) e a Rede Hospitalar, e buscam, junto a essas, formarem uma rede organizada de atenção às urgências, onde a gravidade do caso determina a rapidez com que o paciente será atendido e não a ordem de chegada (BRASIL, 2011).

Essas Unidades são, essencialmente, hospitais simplificados, que buscam reduzir as filas dos prontos atendimentos hospitalares, funcionando 24 horas por dia e sete dias na semana e são aptas a resolver grande parte das urgências e emergências. Além disso, as UPAs devem organizar-se com a Atenção Básica, o SAMU 192, a Atenção Domiciliar e a Atenção Hospitalar regulados pelas Centrais de Regulação de Urgência e complexos reguladores instalados nas regiões de saúde (BRASIL, 2017).

Com isso, é válido lembrar, que as UPAs têm como objetivo prestar atendimento resolutivo e qualificado aos acometidos por quadros crônicos agudizados ou agudos de natureza clínica e prestar o atendimento aos casos de natureza cirúrgica e de trauma. Dessa forma, é de qualificação das Unidades de Pronto Atendimento estabilizar esses pacientes e, também, realizar investigação diagnóstica inicial de modo a estabelecer a conduta primária, assim como oferecer o encaminhamento dos pacientes que necessitarem de atendimentos especializados (BRASIL, 2017).

Porém, as Unidades de Pronto Atendimento têm se tornado uma porta de entrada aos usuários com queixas crônicas e sociais, que buscam esses serviços com a finalidade de resolubilidade para necessidades que deveriam ser atendidas em outros níveis de atenção à saúde, sobrecarregando assim as equipes multiprofissionais (GARCIA e REIS, 2014).

O objetivo desse estudo foi caracterizar a demanda e o tipo de atendimento com base em análise de dados secundários e torna-se relevante a medida que proporcione informações para o planejamento, monitoramento e possível (re)estruturação dos serviços de saúde do município.

\section{METODOLOGIA}

Trata-se de estudo descritivo-observacional, realizado de maneira retrospectiva, com abordagem quantitativa, desenvolvido com dados de uma Unidade de Pronto Atendimento (UPA) do município de Cascavel-PR. A população do estudo foi constituída por usuários maiores de 18 anos de idade, de ambos os sexos, que foram atendidos na UPA Jardim Veneza do referido município, no período de 01/05/2019 a 31/05/2019, e foram classificados de acordo com Protocolo de Manchester. O Sistema de Triagem de Manchester determina uma padronização do atendimento das emergências ao designar a necessidade de preferência ao atendimento a partir da 
indicação clínica, tendo como principal objetivo o estabelecimento de prioridades, onde se levam em conta fatores como: problema do paciente, história clínica, sinais e sintomas, sinais vitais e a condição física (TEIXEIRA, OSELAME, NEVES, 2014).

Dessa maneira os pacientes são classificados por prioridade, mais especificamente em 5 níveis de prioridades: Pacientes com demandas emergentes de cuidado e que precisam de avaliação médica imediata - são classificados no nível 1 (vermelho); Pacientes com demandas muito urgentes e que precisam de atendimento em até 10 minutos - são classificados no nível 2 (laranja); Pacientes com demandas urgentes que devem ser atendidos em até 60 minutos - são classificados no nível 3 (amarelo); Pacientes com problemas pouco urgentes que podem ser atendidos em até 120 minutos - são classificados no nível 4 (verde); Pacientes que não possuem demandas urgentes, e que podem aguardar até 240 minutos para atendimento - são classificados nível 5 (azul) (COUTINHO, CECÍLIO, MOTA, 2012).

Os dados analisados foram fornecidos pela Secretaria Municipal de Saúde por meio do Software-IPM e as variáveis observadas foram: faixa-etária, sexo, distribuição dos atendimentos por cor - protocolo de Manchester, período do atendimento e dia da semana. Foram analisados também, pacientes trazidos via SIATE e via SAMU e suas classificações de risco. Foram excluídos do estudo aqueles com fichas de atendimento da cor azul, pois essas, na unidade estudada, caracterizam fichas de pacientes reavaliados. Exclusos também, pacientes não atendidos, menores de 18 anos, e aqueles que não atenderam aos critérios de inclusão acima descritos.

Os dados coletados foram tabulados em planilha do Excel e analisados por meio de estatística descritiva e os resultados foram apresentados sob forma de tabelas e figuras.

Também foram analisados dados secundários previamente tabulados, fornecidos pelo Serviço de Arquivo Médico e Estatístico (SAME), disponibilizados pela UPA Jardim Veneza e pela Secretaria Municipal de Saúde de Cascavel. Analisou-se a quantidade de pacientes internados, pacientes que necessitaram de suporte, quantidade e tipo de procedimentos realizados, sendo esses, suturas, curativos, drenagem de abscesso, exérese de corpo estranho, sondagem de demora, sondagem de alívio, sondagem gástrica, medicações administradas e procedimentos injetáveis.

Verificou-se também, o manejo e desfecho dos pacientes, entre esses, internamento, reavaliação, observação de acordo com tempo de permanência, evasão, alta a pedido, pacientes encaminhados a hospitais da cidade de Cascavel (Centro de Oncologia de Cascavel (CEONC), União Oeste Paranaense de Estudos e Combate ao Câncer (UOPECCAN), Hospital São Lucas (HSL), Hospital Universitário do Oeste do Paraná (HUOP), Hospital Nossa Senhora da Salete (HNSS)), pacientes encaminhados a outros hospitais localizados fora do município de Cascavel e óbitos.

Este trabalho, por se tratar de pesquisa com seres humanos, está em cumprimento com a Resolução 466/12 do Conselho Nacional de Saúde. A pesquisa foi aprovada pelo Comitê de Ética em Pesquisa do Centro Universitário Fundação Assis Gurgacz com o Certificado de Apresentação para Apreciação Ética (CAAE) de número 18712219.7.0000.5219.

\section{RESULTADOS E DISCUSSÃO}

\subsection{ATENDIMENTO POR PROCURA ESPONTÂNEA}

No período de maio de 2019 foram registrados 10.834 dados no Sistema - IPM. Foram excluídos 3.361 atendimentos realizados pela UPA Pediátrica, pois esses não FAG Journal of Health - ISSN 2674-550X, 2020, v.2, n.1, p. 58 
englobavam o objetivo do trabalho. $\mathrm{O}$ atendimento por procura espontânea, inclui os pacientes que são atendidos em consultório médico e os pacientes que por certas vezes, são encaminhados diretamente a sala de procedimentos como nos casos de suturas e exéreses de corpos estranhos. A procura espontânea pelo serviço foi de 6.813 pacientes, sendo que apenas 6.172 desses foram atendidos, 641 pacientes passaram pela triagem da enfermagem, porém, evadiram-se antes do atendimento ser concluído. Entre os 6.172 pacientes atendidos, foram excluídos 288 sendo classificados segundo o protocolo de Manchester como fichas azuis, pois esses, na unidade avaliada, caracterizam pacientes que foram reavaliados. Além disso, foram excluídos os pacientes menores de 18 anos, correspondendo a 401 pacientes.

Portanto, a amostra válida para o presente trabalho foi de 5.483 pacientes que realizaram a procura espontânea pela UPA Jardim Veneza no mês de maio de 2019.

Quanto ao sexo, $2.955(53,9 \%)$ dos usuários que procuraram a UPA no período estudado eram do sexo feminino e $2.528(46,1 \%)$, do masculino. Tais dados são semelhantes aos obtidos por Oliveira et al. (2011) em que 53,7\% dos usuários de seu estudo pertenciam ao sexo feminino e $46,3 \%$ do masculino.

Quanto à faixa etária, indivíduos jovens com idade entre 21 a 30 anos foram os que mais procuraram o serviço de pronto atendimento sendo 1.760 correspondendo a $32 \%$ dos atendimentos. Seguidos pelos indivíduos adultos entre 31 a 40 anos, que corresponderam a 1.137 pacientes $(20,7 \%)$, conforme mostra a Tabela 1.

Tabela 1. Distribuição conforme sexo e faixa etária dos pacientes que procuraram a UPA - Jardim Veneza.

\begin{tabular}{ccc}
\hline & $\mathbf{1 0 0} \%(\mathbf{N}=\mathbf{5 . 4 8 3})$ & \\
\hline CARACTERíSTICA & $\mathrm{N}($ total 5.483) & \\
Sexo & & \\
Feminino & 2.955 & $53,9 \%$ \\
Masculino & 2.528 & $46,1 \%$ \\
Faixa Etária & & \\
$18-20$ & 531 & $9,60 \%$ \\
$21-30$ & 1.760 & $32,00 \%$ \\
$31-40$ & 1.137 & $20,70 \%$ \\
$41-50$ & 795 & $14,40 \%$ \\
$51-59$ & 535 & $9,67 \%$ \\
$>$ ou $=60$ & 725 & $13,20 \%$ \\
\hline
\end{tabular}

Quanto à classificação, segundo o protocolo de Manchester, os pacientes poucos urgentes foram predominantes, correspondendo a 3.862 atendimentos $(70,2 \%)$ classificados com a cor verde. No Brasil a prevalência do uso inadequado dos serviços de pronto atendimento variou de $39 \%$ no estudo de Stein (2002) e $81,7 \%$ no estudo de Amarante (2014). Estudos realizados em serviços de urgência internacionais verificaram também alta prevalência desse problema; Backman et al. (2008) estimaram que mais de $55 \%$ das visitas aos serviços de emergência nos EUA e Europa foram por problemas não urgentes.

A alta prevalência do uso errôneo dos serviços de urgência é alarmante, uma vez que eleva as despesas do sistema de saúde, aumenta a demanda dos serviços de urgência com atendimentos que poderiam ter resolutividade em outros níveis de atenção e que, de certo modo, competem com casos realmente urgentes. As consultas, nas unidades de pronto atendimento, visam alívio rápido dos sintomas dos pacientes, não havendo vínculo com profissionais de saúde, sem garantia de prevenção de complicações e novas doenças (AMARANTE, 2014).

Dessa maneira, a demanda excessiva nos serviços de urgência/emergência é devido a maioria dos atendimentos ser decorrente de problemas de baixa complexidade que poderiam ser resolvidos em serviços de atenção básica ou FAG Journal of Health - ISSN 2674-550X, 2020, v.2, n.1, p. 59 
especializados. Esse perfil de demanda configura uma das principais portas de entrada ao sistema de saúde e, possivelmente, revela, entre outras coisas, distribuição desigual da oferta de serviços, não apenas do ponto de vista quantitativo, mas, também qualitativo na atenção básica, especializada e também na hospitalar (OLIVEIRA et al, 2011).

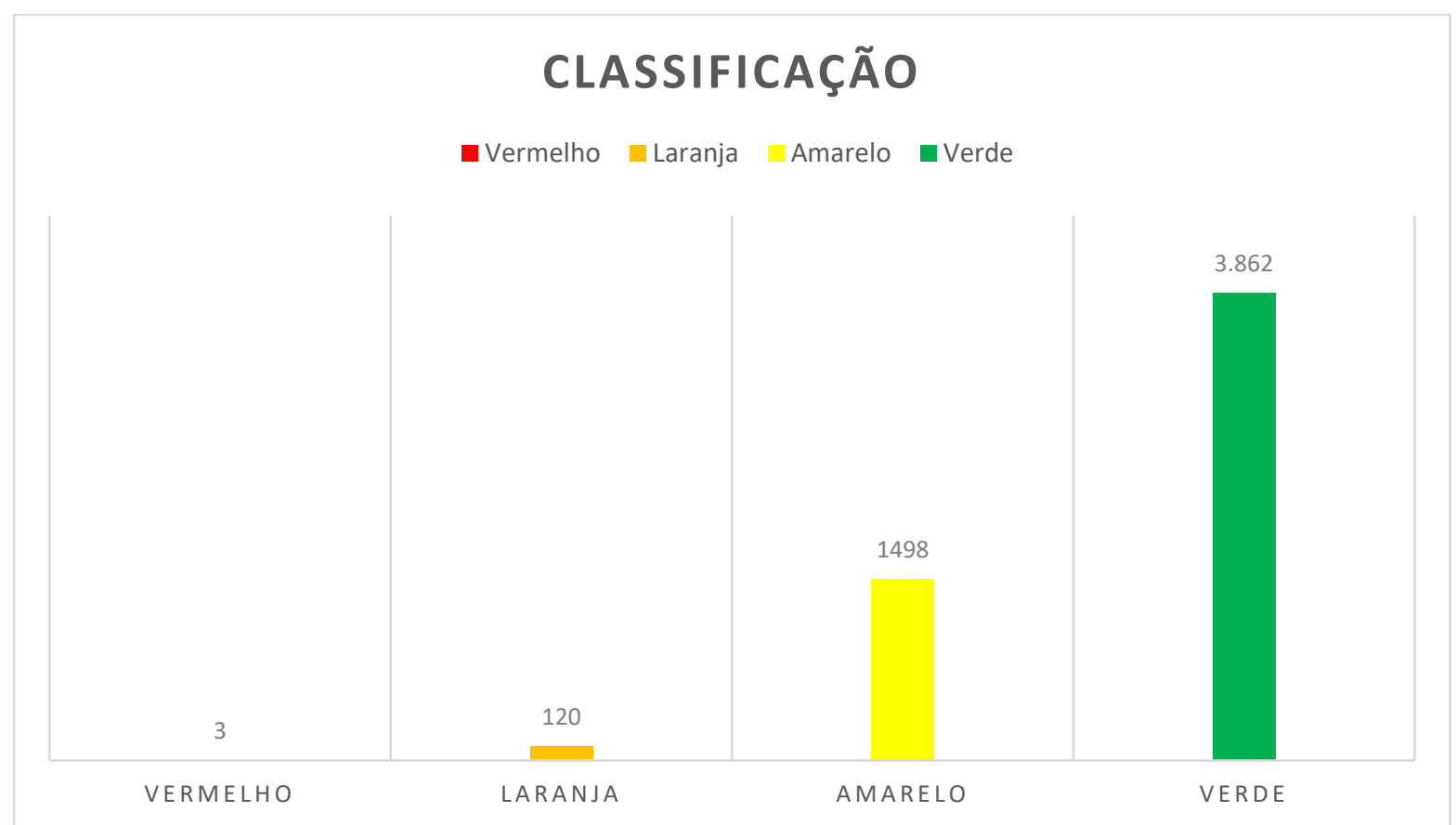

Figura 1. Distribuição conforme classificação de cor dos pacientes atendidos no mês de maio de 2019 na UPA Jardim Veneza de Cascavel-PR.

Com relação aos dias da semana, verificou-se que 4.344 (79,22\%) buscaram o serviço de pronto atendimento nos dias úteis da semana, e em horário em que as UBSs ou a USF estão funcionando e que, a priori, deveriam absorver essa demanda.

A procura pelo serviço, durante o final de semana, ocorreu apenas em 1.139 $(20,78 \%)$ atendimentos e o domingo foi o dia de menor utilização do serviço, com 544 $(9,92 \%)$ pacientes. Os dias da semana de maior procura foram as quintas 1.002 $(18,27 \%)$ e as quartas feiras 907 (16,54\%). O mesmo foi observado em relação aos horários de atendimento, pois $3.103(56,60 \%)$ atendimentos ocorreram no período das 8:00 da manhã às 17:59 da tarde, horários em que as UBSs e as USFs encontram-se em funcionamento. O período noturno entre 18:00 a 23:59h correspondeu a 1.469 $(26,80 \%)$ dos atendimentos, e a madrugada da 00:00 às 7:59 da manhã foi o período de menor procura, apenas 911 (16,60\%) usuários procuraram a unidade nesse período.

De acordo com Garcia e Reis (2014), a procura por um serviço de pronto atendimento pode ser atribuída ao fato de que a lógica do serviço nem sempre é a mesma da população. A demora no agendamento das consultas da UBS, os horários de funcionamento coincidindo com os turnos de trabalho e a obtenção de medicações facilitadas no PA resultam em uma maior procura por esse tipo de atendimento.

Para Oliveira et al. (2011), embora as unidades de saúde da rede fiquem abertas, o modelo hospitalocêntrico ainda é o vigente no país. Justifica-se assim a procura do pronto atendimento ocorrer majoritariamente no período em que as UBSs e USFs encontram-se funcionando, ademais, a demanda de queixas não urgentes que 
poderiam ser resolvidas na UBS ou USF foi predominante. Os dados analisados estão relacionados na Tabela 2.

Tabela 2. Distribuição dos usuários adultos atendidos durante mês de maio na UPA Jardim Veneza, segundo dias da semana e período de atendimento.

\begin{tabular}{ccc}
\hline & $\mathbf{1 0 0} \%(\mathbf{N}=\mathbf{5 . 4 8 3})$ & \\
\hline CARACTERístICA & $\mathrm{N}($ total 5.483$)$ & \\
Dias da Semana & & $100)$ \\
Segunda-feira & 855 & $15,59 \%$ \\
Terça-feira & 706 & $12,87 \%$ \\
Quarta-feira & 907 & $16,54 \%$ \\
Quinta-feira & 1.002 & $18,27 \%$ \\
Sexta-feira & 875 & $15,95 \%$ \\
Sábado & 595 & $10,86 \%$ \\
Domingo & 544 & $9,92 \%$ \\
Dias úteis & 4.344 & $79,22 \%$ \\
Final de semana & 1.139 & $20,78 \%$ \\
Períodos de atendimento & & $16,60 \%$ \\
8:00 às 17:59 & 3.103 & $56,60 \%$ \\
18:00 às 23:59 & 1.469 & $26,80 \%$ \\
\hline 00 às 7:59 & 911 & \\
\hline
\end{tabular}

\subsection{ATENDIMENTOS DE AMBULÂNCIAS E PROCEDIMENTOS REALIZADOS}

\subsubsection{ATENDIMENTO VIA SAMU}

O total de usuários que foram atendidos na UPA Jardim Veneza durante o mês de maio, trazidos via SAMU, foi de 231 pacientes. Desse total, a maioria, $142(61,47 \%)$ pacientes, foram classificados como prioridade de cor amarela. Seguidos por 63 $(27,27 \%)$ pacientes considerados nível de prioridade verde, e apenas $20(8,65 \%)$ pacientes foram classificados como prioridade laranja, um número menor ainda, 3 $(1,29 \%)$ pacientes foram considerados nível de prioridade vermelho.

\subsubsection{ATENDIMENTO VIA SIATE}

O total de usuários que chegaram a UPA Jardim Veneza, trazidos pelo SIATE, foi de 77 pacientes. Desses, a maioria, $56(72,72 \%)$ usuários também foram classificados como prioridade amarela. Seguidos pelos 18 (23,37\%) usuários classificados como prioridade verde. Nenhum indivíduo com prioridade vermelha foi levado via SIATE à Unidade de Pronto Atendimento Jardim Veneza durante o mês de maio.

\subsubsection{CLASSIFICAÇÃO DOS PROCEDIMENTOS REALIZADOS NA UPA}

Os dados a seguir referem-se aos usuários que procuraram de forma espontânea a Unidade de Pronto Atendimento e foram encaminhados diretamente a 
sala de procedimentos para realização de curativos, suturas, drenagem de abscessos, exérese de corpo estranho, entre outros procedimentos.

O total da procura, para esses fins, foi de 264 pacientes. Desses, $160(60,60 \%)$ usuários foram classificados com prioridade verde, $89(33,71 \%)$ pacientes com prioridade amarela, 9 (3,41\%) pacientes classificados como azuis e $6(2,28 \%)$ usuários com prioridade laranja. Nenhum indivíduo com prioridade vermelha buscou a Unidade de Pronto Atendimento para a realização de procedimentos.

\section{Distribuição conforme classificação por cor}

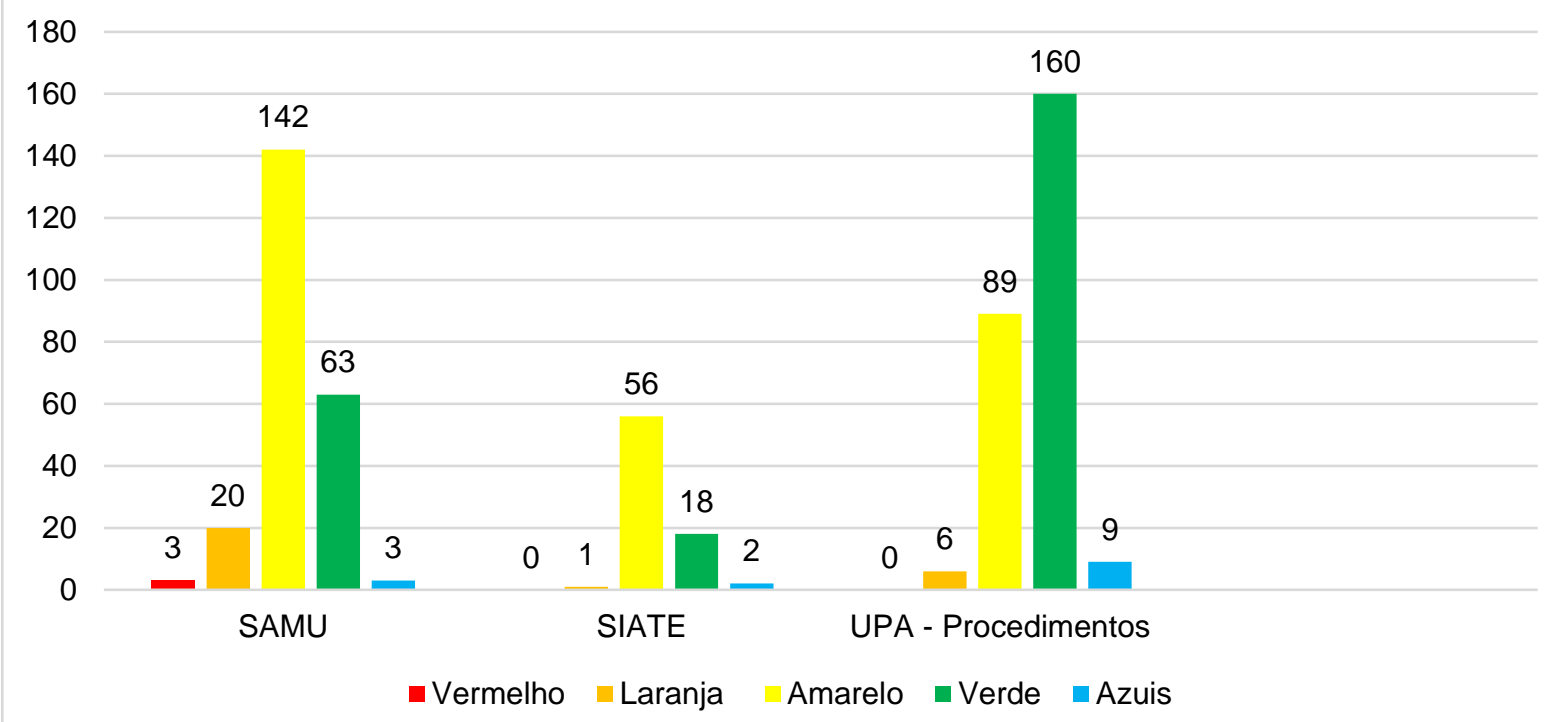

Figura 2. Dados referentes à distribuição dos atendimentos conforme classificação por cor, realizados na UPA Veneza, provenientes via SAMU, SIATE e apenas para realização de Procedimentos.

\subsection{PROCEDIMENTOS E MANEJO DOS PACIENTES}

As UPAs devem oferecer atendimento resolutivo e qualificado aos acometidos por quadros agudizados ou agudos de natureza clínica e prestar o atendimento aos casos de natureza cirúrgica e de trauma. Dessa forma, é atributo das Unidades de Pronto Atendimento estabilizar esses pacientes e, além disso, executar uma investigação diagnóstica inicial com a finalidade de indicar a conduta primária, e igualmente, garantir o encaminhamento aos usuários que necessitarem de atendimentos especializados (BRASIL, 2017).

\subsubsection{TOTALIDADE DE PROCEDIMENTOS REALIZADOS}

Foi realizado levantamento de todos os procedimentos realizados na UPA Jardim Veneza durante o mês de maio de 2019, desde os pacientes na triagem ao manejo dos pacientes internados. Dentre os procedimentos os mais realizados são suturas, curativos, drenagem de abscessos, exérese de corpo estranho, sondagem de demora, sondagem de alívio, sondagem gástrica, medicamentos administrados via oral e medicamentos administrados de forma injetável, tanto intramuscular profunda quanto endovenosa. $O$ procedimento mais prevalente na referida unidade foi o de medicamentos administrados via injetável, totalizando 10.898 procedimentos injetáveis. Tal dado assemelha-se ao obtido por Garcia e Reis (2014) e por Oliveira et al. (2011) onde o número de procedimentos injetáveis endovenosos e intramusculares também foram os mais prevalentes. Os dados analisados estão relacionados na figura a seguir. 
Procedimentos realizados durante mês de maio de 2019 na UPA Jardim Veneza

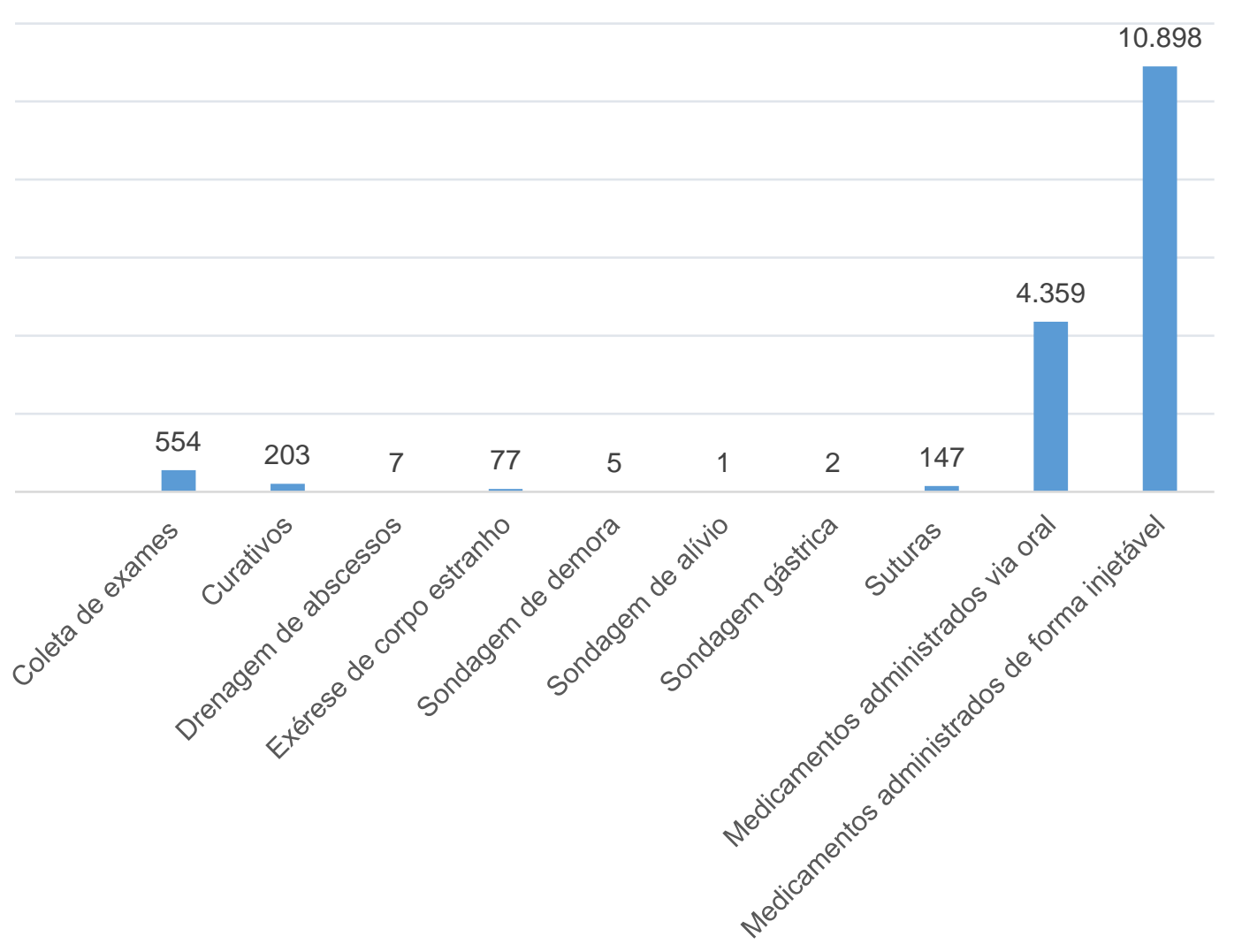

Figura 3. Distribuição dos procedimentos realizados na UPA Jardim Veneza no mês de maio de 2019.

\subsubsection{MANEJO E DESFECHO DOS PACIENTES}

Em relação ao manejo dos pacientes, analisaram-se pacientes que permaneceram em observação por menos de 6 horas, observação por menos de 12 horas e em observação por mais de 12 horas, pacientes que necessitaram de internamento, pacientes que foram reavaliados, pacientes reavaliados por outros profissionais, altas e óbitos.

Entre os desfechos dos pacientes, foram analisados os usuários que se evadiram após internamento, usuários que obtiveram alta a pedido, pacientes encaminhados aos hospitais do município de Cascavel sendo esses: Hospital São Lucas, Hospital Universitário do Oeste Paranaense, Hospital Nossa Senhora Salete, Hospital UOPECCAN e além disso analisaram-se os encaminhamentos à outros hospitais localizados em outros municípios.

\section{CONSIDERAÇÕES FINAIS}

A análise das informações obtidas com essa pesquisa permitiu evidenciar que, a maioria dos usuários procurou espontaneamente UPA Jardim Veneza, sendo esses usuários majoritariamente do sexo feminino, jovens, na faixa etária dos 21 aos 30 anos, e que possuem queixas pouco urgentes que, em sua maioria, poderiam ser 
resolvidas em uma Unidade Básica de Saúde. Constatou-se também que o horário de maior demanda é o mesmo em que as UBSs encontram-se funcionando, acredita-se que tal resultado deve-se ao fácil acesso e à resolubilidade fornecida pela unidade de pronto atendimento. Percebeu-se que sendo as UPAs, serviços que atendem com as portas abertas, realizando atendimento 24 horas e considerando que há dificuldade de acesso à consulta nas Unidades Básicas de Saúde e tendo em vista a agilidade na solução dos problemas de saúde grande parte da população acaba buscando atendimento nessas unidades, até mesmo quando o problema de saúde não representa grande complexidade.

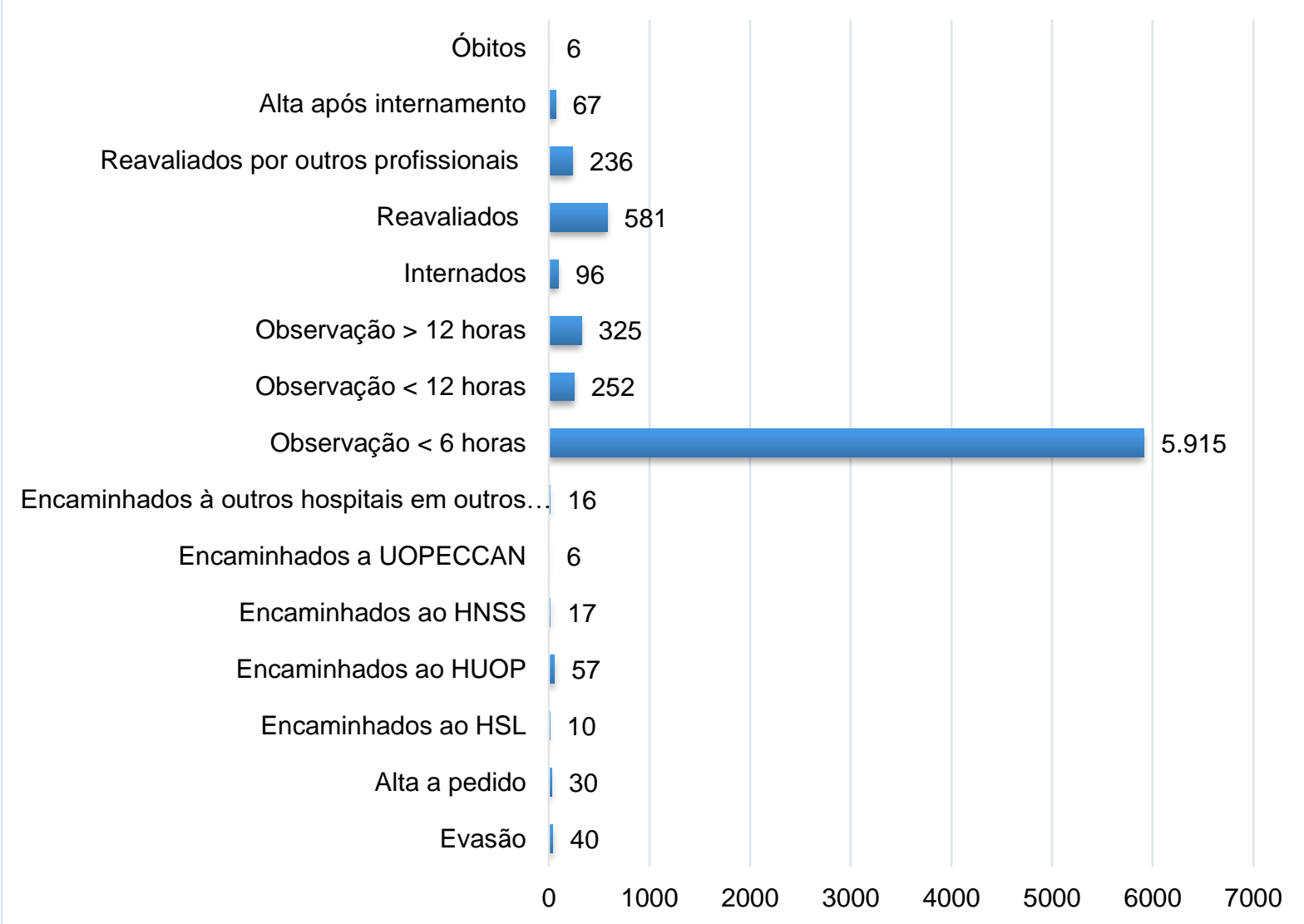

Figura 4. Manejo e desfecho dos pacientes que procuraram a UPA Jardim Veneza no mês de maio de 2019.

Desse modo, os dados coletados confirmam a hipótese desse estudo de que a UPA realiza atendimentos que não se relacionam diretamente com sua proposta inicial e paralelamente, confirma as pesquisas realizadas por Lovalho (2007), Furtado (2004) e Costa, Wong e Barbosa (2012), que apontam que a maioria dos atendimentos realizados pelas UPAs é de baixa complexidade, o que não condiz com a proposta apresentada pelo sistema.

Entre os pacientes que chegam à unidade por demandas não espontâneas, sendo esses, trazidos via SAMU e SIATE, os atendimentos de média complexidade, classificados com a cor amarela foram preponderantes.

Constatou-se também que muitos usuários procuram a UPA para a realização de procedimentos, esses, predominantemente, de baixa complexidade e pouco urgentes, passíveis de realização nas Unidades Básicas de Saúde, como troca de curativos.

Entre todos os procedimentos realizados, tanto pela enfermagem tanto por médicos, têm-se um número exponencial de 10.898 procedimentos injetáveis, 
seguidos por 4.359 medicações administradas. Tais números caracterizaram alta demanda do sistema.

Concluiu-se que esse tipo de estudo é de fundamental importância para caracterizar a demanda da população que procura o serviço de pronto atendimento, e delinear o perfil dos pacientes, para que usos inadequados do serviço sejam reconhecidos e minimizados, a fim de evitar superlotação dos serviços. Sugere-se, portanto, uma ampliação da cobertura das unidades básicas (UBS + USF), junto a uma melhoria no atendimento e na resolubilidade dos serviços, uma vez que caracterizar a demanda e o perfil dos pacientes, oportuniza o desenvolvimento de campanhas e políticas educacionais direcionadas à população cascavelense, com o intuito de informar sobre o uso correto dos serviços de saúde.

\section{REFERÊNCIAS}

AMARANTE, L. C. D. S. Caracterização da demanda dos serviços de saúde em unidades de pronto atendimento segundo critérios classificação de risco, características sócio demográficas e super utilização. Piracicaba, 2014. 82 f. (Tese Curso de Odontologia), Universidade Estadual de Campinas (UNICAMP). Disponível em: <http://repositorio.unicamp.br/jspui/bitstream/REPOSIP/290870/1/Amarante_Lidiane CintiadeSouza_M.pdf>. Acesso em: 19 nov. 2019.

BRACKMAN A.S.; BLOMGVIS T. P.; LAGERLUND M.; HOME E.C.; ADAMI J. Characteristics of non-urgent patients. Scand J Prim Health Care. 2008; 26(3): 181-7.Disponível em: <https://emj.bmj.com/content/29/12/1004>. Acesso em: 19 nov. 2019. http://dx.doi.org/10.1136/emermed-2011-200464

BRASIL, Ministério da Saúde. Portaria №. 1600, de 07 de julho de 2011. Reformula a Política Nacional de Atenção às Urgências e institui a Rede de Atenção às Urgências no Sistema Único de Saúde. Diário Oficial da União 08 jul 2011; Seção 1. Disponível em: <http://bvsms.saude.gov.br/bvs/saudelegis/gm/2011/prt1600_07_07_2011.html>. Acesso em: 19 nov. 2019.

BRASIL, Ministério da Saúde. Portaria n‥ 10, de 3 de janeiro de 2017. Redefine as diretrizes de modelo assistencial e financiamento de UPA 24h de Pronto Atendimento como Componente da Rede de Atenção às Urgências, no âmbito do Sistema Único de Saúde. 2017. Ministério da Saúde. Disponível em: <http://bvsms.saude.gov.br/bvs/saudelegis/gm/2017/prt0010_03_01_2017.html>. Acesso em: 19 nov. 2019.

CASSETTARI, S. S. R.; DE MELLO, A. L. S. F. Demanda e tipo de atendimento realizado em Unidades de Pronto Atendimento do município de FLORIANÓPOLIS, Brasil: Texto \& Contexto Enfermagem, v. 26.1, p.1-9, 2017. Disponível em: < http://www.scielo.br/pdf/tce/v26n1/pt 1980-265X-tce-26-01-e3400015.pdf>. Acesso em: 19 nov. 2019. http://dx.doi.org/10.1590/0104-07072017003400015

COSTA, J. S. M.; WONG, L. R.; BARBOSA, A. C. Q. Redes de Atenção às Urgências e Emergências: Análise da Demanda em Três Pontos Fixos de Assistência à Saúde, Estudo de Caso no Distrito Sanitário de Venda Nova, Município de Venda Nova. XV Seminário sobre a Economia Mineira, Cedeplar, 2012. 
COUTINHO, A. A. P.; CECÍLIO, L. C. D. O; MOTA, J.A. C. Classificação de risco em serviços de emergência: uma discussão da literatura sobre o Sistema de Triagem de Manchester. Revista Médica de Minas Gerais-Rmmg, v. 22, n. 2, 2012.

FURTADO, B. M. A. S. M; ARAÚDO JR., J. L. C; CAVALCANTI, P. O perfil da emergência do Hospital da Restauração: uma análise dos possíveis impactos após $O$ a municipalização dos serviços de saúde. Revista Brasileira de Epidemiologia, São Paulo, v. 7, n. 3, p. 279-289, set. 2004. http://dx.doi.org/10.1590/S1415$\underline{790 \times 2004000300006}$

GARCIA, V. M.; REIS, R. K. Perfil de usuários atendidos em uma unidade não hospitalar de urgência. Revista Brasileira de Enfermagem, Riberão Preto, v. 67, ed. 2, 2014. Disponível em: <http://www.scielo.br/pdf/reben/v67n2/0034-7167-reben-6702-0261.pdf.> Acesso em: 19 nov. 2019. http://dx.doi.org/10.5935/0034$\underline{7167.20140035}$

HERNANDEZ, P. F. Unidade de Pronto Atendimento e a articulação com os níveis de atenção às urgências e emergências. Revista Políticas Públicas e Desenvolvimento, $\quad$ v. $1, \quad$ n. 1,2018 . Disponível em: < http://www.ichs.uff.br/ojs/index.php/rppd/article/viewFile/14/4 >. Acesso em: 19 nov. 2019.

LOVALHO A.F. Administração de serviços de saúde em urgências e emergências. 0 mundo da Saúde, São Paulo, v.28,n.2, p.160-171, abr./jun.2007.

OLIVEIRA G.N.; SILVA M.F.N.; ARAUJO I.E.M.; CARVALHO M.A.F. Perfil da população atendida em uma unidade de emergência referenciada. Rev. Latino-Am. Enfermagem. Maio-jun. 2011. ;19(3): 09 telas. Disponível em: < http://www.scielo.br/pdf/rlae/v19n3/pt $14>$. Acesso em: 19 nov. 2019. http://dx.doi.org/10.1590/S0104-11692011000300014

STEIN A.T.; HARZHEIM E.; COSTA M.; BUSNELLO E.; RODRIGUES L.C;. The relevance of continuity of care: a solution for the chaos in the emergency services. Fam Pract 2002;19(2):207-10. https://doi.org/10.1093/fampra/19.2.207

TEIXEIRA, V.D.A.; OSELAME, G.B.; NEVES, E.B. O Protocolo de Manchester no sistema único de saúde e a atuação do enfermeiro. Revista da Universidade Vale do Rio Verde, v. 12, n. 2, p. 905-920, 2014. Disponível em: < http://periodicos.unincor.br/index.php/revistaunincor/article/view/1769/pdf 268 > Acesso em: 19 nov. 2019. http://dx.doi.org/10.5892/ruvrd.v12i2.1769 